\title{
New Developments in Local Area Helioseismology
}

T. L. Duvall, Jr.

Laboratory for Astronomy and Solar Physics, NASA/GSFC, USA

\author{
A. G. Kosovichev \\ W.W.Hansen Experimental Physics Laboratory, Stanford University, \\ Stanford, CA, USA
}

\begin{abstract}
.
Several techniques are used to study local areas in helioseismology, including time-distance helioseismology, acoustic imaging/holography, and ring diagram analysis. These techniques can be used to study flows, magnetic fields, and temperature inhomogeneities. The "local" area studied can be as small as a supergranule, or as large as the entire convection zone in the case of meridional circulation as studied by Giles and colleagues. Active regions have been studied with some interesting results, with complicated flow patterns below sunspots and detectable sound speed inhomogeneities in the $10 \mathrm{Mm}$ below the spots. Another interesting result is the detection of sunspots on the back side of the Sun by Lindsey and Braun using the holography technique. A confirmation of their result using the time-distance technique is presented.
\end{abstract}

\section{Introduction}

One of the primary objectives of the SOHO mission is to study the structure and dynamics of the internal structure of the Sun. Methods of local-area helioseismology which are being intensively developed provide 3-dimensional images of the interior. These methods require high stability and low-noise series of Doppler images in order to measure local propagation properties (travel times, phases, amplitudes) of solar waves. The MDI instrument on SOHO has provided such unique data of various spatial resolution, which has been used to obtain new insight into the physics of the internal processes. In this article we review some recent results obtained only by one method, time-distance helioseismology, which represents a leading direction in the local-area diagnostics. We give four examples of the new developments which are based on the SOHO/MDI of various resolution. First, we present new results of imaging the structure of sunspots, obtained from relatively short, typically 8 hours long, time series of high-resolution MDI images (0.6 arcsec per pixel). Then, we discuss results from full-disk MDI data with 2 arcsec/pixel resolution on mapping near-surface largescale flows and structure associated with active regions on both the front and far sides of the Sun. Finally, we demonstrate how the medium-resolution data (10 arcsec/pixel) continuously downlinked from SOHO are used to probe deep 

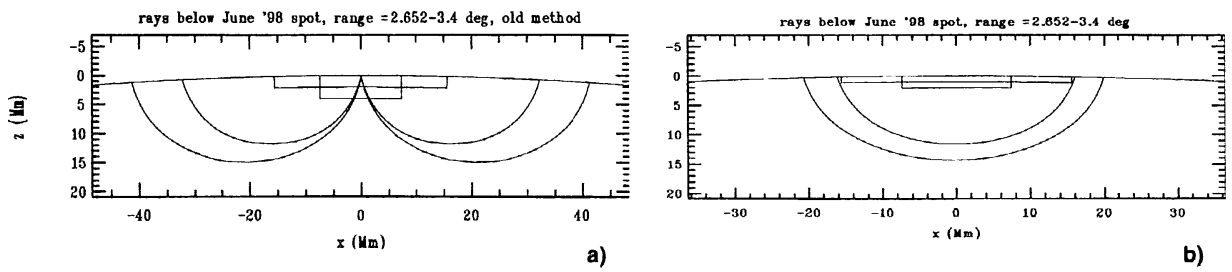

Figure 1. Illustration of two types of time-distance measurements: a) "surface-focusing" scheme, b) "deep-focusing scheme". The curves show typical ray paths of acoustic waves. The first method measures the travel times for waves propagating between a central point and wide annuli surrounding this point. In the second method the travel times are measured between points on the opposite sides of the central point.

zonal and meridional flows. For recent reviews of other methods and results of local-area helioseismology we refer the reader to the Topical Issue "Helioseismic Diagnostics of Solar Activity and Convection" of Solar Physics, vol. 192, 2000.

\section{Looking Beneath Sunspots}

Travel times of waves propagating through the solar interior are measured by cross-correlating oscillating signals observed at different points on the surface (Duvall et al, 1993). Because of the stochastic nature of solar oscillation it is generally required to average the cross-correlation for a particular range of distances both over some spatial areas and in time in order to accumulate a good signal-to-noise ratio. Two typical schemes of the spatial averaging are shown in Figure 1. For the so-called "surface-focusing" scheme (Fig.1a) the measured travel times are mostly sensitive to the near surface condition at the central point where the ray paths are focused. However, by measuring the travel times for several distances and applying an inversion procedure it is possible to infer the distribution of the variations of the wave speed and flow velocities with depth (Kosovichev \& Duvall, 1997). The averaging can done in such a way that the "focus" point is located beneath the surface (Duvall, 1995). An example of the "deep-focusing" scheme is shown in Fig. 1b. In this case the travel times are more sensitive to deep structures but still inversions are required for correct interpretation. First results show that "deep-focusing" allows us to see more features in the interior.

We have used both of the schemes for measuring variations of the wave speed beneath a sunspot. In this case the second scheme has an advantage that the measurement (cross-correlation) points are completely outside the spot. Both schemes provide qualitatively similar results (Fig. 2). However, "deep-focusing" seems to reveal more details. Beneath the sunspot we find a lower wave speed region extending down to $4 \mathrm{Mm}$ in depth, and then a rather sharp transition to higher wave speeds which is clearly seen down to $16 \mathrm{Mm}$. The non-uniform structure is surprising, and currently it is not understood whether the variations of the wave speed are due to magnetic fields or accumulated heat flux or both. 


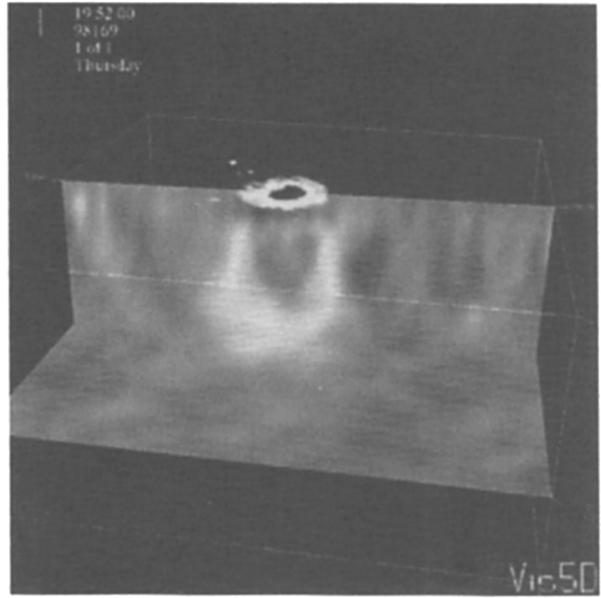

a)

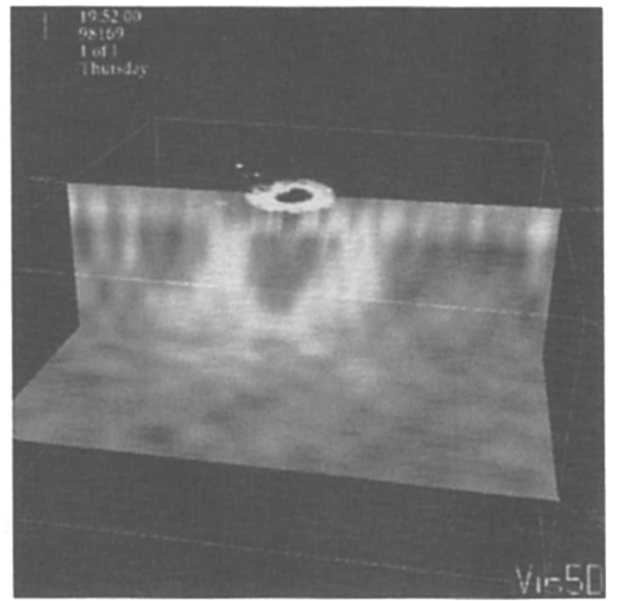

b)

Figure 2. The sound-speed perturbation in a sunspot obtained using the "surface-focusing" (a) and "deep-focusing" (b) schemes. The horizontal size of the box is 13 degrees $(158 \mathrm{Mm})$, the depth is $24 \mathrm{Mm}$. The positive variations are shown in red, and the negative ones in blue.

Recent attempts to measure anisotropy of the wave speed which can provide a measure of the magnetic field strength have not been conclusive. However, the velocity field beneath the sunspot has been measured. The results reveal a complicated pattern which consists of vortex flows converging beneath the spot in accordance with Parker's (1993) model, and some strong directed flows which are probably related to formation of pores linked to the spot (Zhao, et al, 2001).

\section{Mapping Subsurface Flows and Active Structures}

In general, convective flows must play a major role in the mechanisms of solar activity. Therefore, it is very important for both understanding the physics of solar activity and forecasting to study various aspects of the internal dynamics. Duvall and Gizon (2000) have developed a diagnostic of near-surface layers by applying the time-distance approach to surface gravity waves (f modes). These waves are much less sensitive to temperature variations, and therefore the mean travel-differences for these waves are mostly due to magnetic field. The f-mode waves also provide very accurate measurements for horizontal mass flows just beneath the solar surface, in the upper layer 1-2 Mm thick, and allow us to detect both large-scale and small-scale flow patterns associated with solar activity (Gizon et al, 2000). Figure 3a shows large-scale flow patterns during Carrington Rotation 1948 (April 4, 1999 - May 1, 1999). These results reveal converging large-scale flows associated with active regions, with a velocity of $\simeq 50 \mathrm{~m} / \mathrm{s}$. Figure $3 \mathrm{~b}$ shows the travel-time f-mode signal caused by magnetic fields (top panel) and the corresponding surface magnetic field map (bottom). These results are obtained from the MDI full-disk Dynamics data which are available typically 


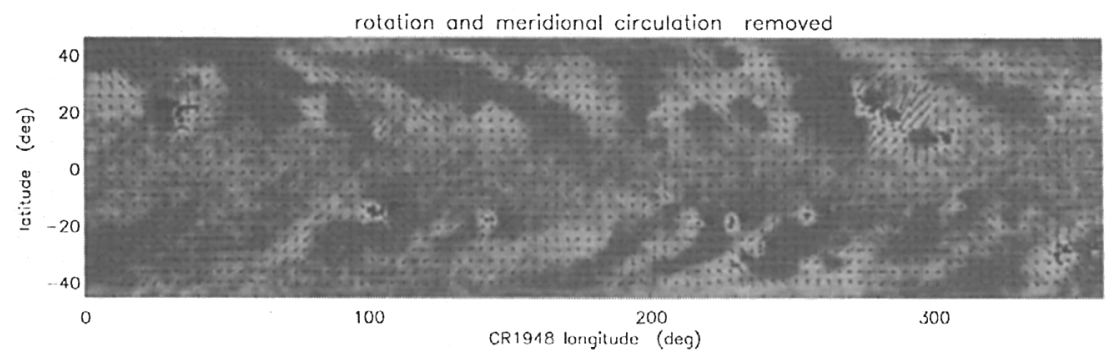

a)

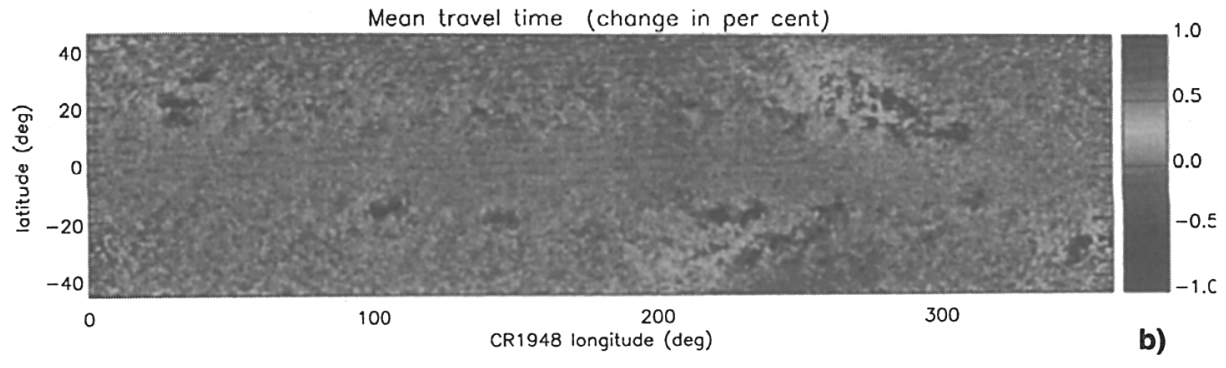

Figure 3. a) Large-scale near-surface flows associated with active regions, obtained from surface gravity waves (f-modes). The background color map shows magnetic field. b) Variations of the f-mode travel times due to magnetic fields in a near-surface layer. (Gizon et al., 2001).

for two months each year. Therefore, currently it is not possible to watch the interaction between the large-scale fields and flows on longer time scales, and determine if by observing the flow patterns one can predict future activity. The f-mode diagnostics are very promising and will receive further development.

\section{Imaging the Far-Side of the Sun}

A new approach for predicting new active regions on the front side of the Sun has been recently demonstrated by Lindsey and Braun (2000). They used a holographic technique to image the far side of the Sun. This allows early detection of new active regions which a few days later will rotate to the front side and affect the space weather. The holographic method is discussed in these Proceedings by Braun and Lindsey. We only mention that the time-distance technique also can provide measurements of the travel-time perturbations on the far side. We illustrate this in Fig. 4, the left panel of which shows the measurement scheme. This is essentially a deep-focusing scheme with the focus point chosen on the far-side surface. The left panel of Fig.4 shows a map of the travel time variations on the far side. The travel time becomes shorter by $\simeq 6 \mathrm{sec}$ when there is an active region in the focus. The far-side signal was recovered from 48-hour time series of medium resolution images. 


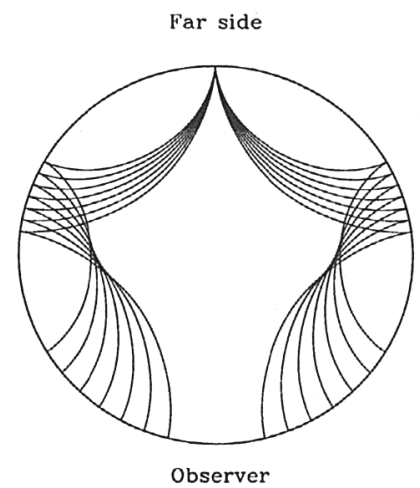

a)

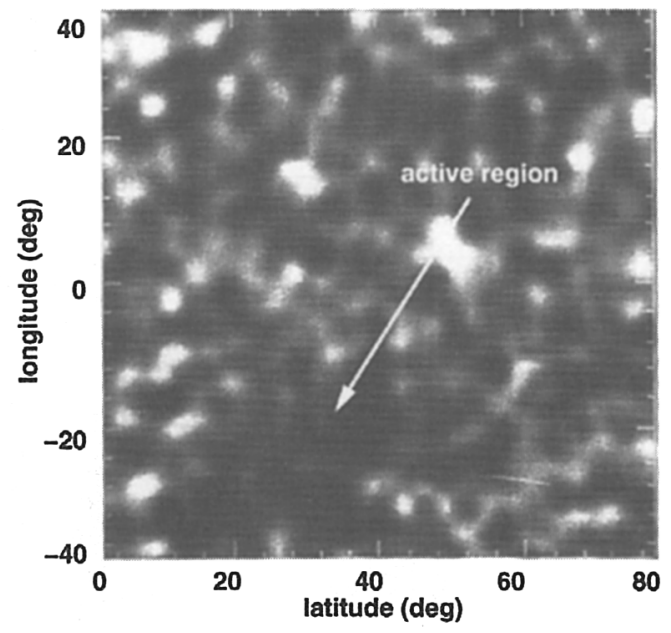

b)

Figure 4. Left panel: A "deep-focusing" scheme with the acoustic ray focus point on the far side of the Sun. Right panel: A map of the travel-time variations (white - positive, and black - negative) due to perturbations on the far-side surface. A dark region at $-20^{\circ}$ longitude and $30^{\circ}$ latitude is caused by shorter travel times due to an active region.

\section{Probing Deep Large-Scale Flows}

Measuring flows and structures in the deep interior requires substantially longer time series of Doppler images. Of particular interest is the tachocline zone located at base of the convection zone at the depth of $200 \mathrm{Mm}$ (0.71 solar radius). This is believed to be the main site of the solar dynamo. However, no reliable information about this zone has been obtained by the local-area seismology methods. However, the recent results by Giles (1999) and colleagues show the potential of the time-distance technique for probing deep flows. Giles applied a version of the "deep-focusing" scheme and a regularized least-squares inversion technique to the MDI medium resolution (Structure) data to determine zonal and meridional flows in the convection zone (Fig. 5a). The spatial resolution drastically decreases with depth. Fig.5b shows a sample of averaging kernels which characterize the averaging properties of the inversion results and provide a measure of the spatial resolution.

Some results of this investigation are presented in Fig. 6 which shows the rotation rate and the speed of the meridional circulation as a function latitude at 6 different depths. The symmetrical with respect to the equator component of the rotation rate has been determined also by standard methods of "global" helioseismology, from rotational splitting of normal mode frequencies of solar oscillations. The results are compared in Fig.6 (left panel), and demonstrate good agreement. This serves as a test of the time-distance approach and provides confidence in the results. The meridional circulation inside a star has been 


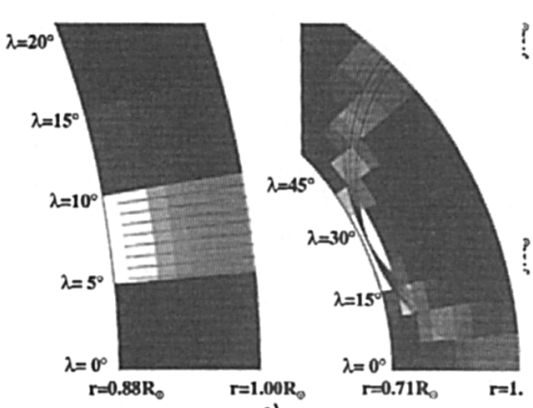

a)
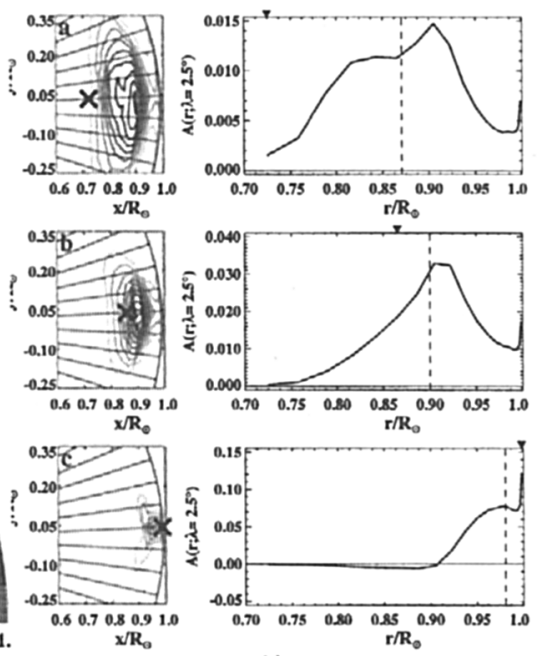

b)

Figure 5. a) the sensitivity kernels for the zonal velocity for $\lambda=$ $7.5^{\circ}, \Delta=18.1^{\circ}$ (left) and for the meridional velocity for $\lambda=24.4^{\circ}$, $\Delta=44.1^{\circ}$ (right). The light colors show the sensitivity to horizontal flows, with dark areas being those with no sensitivity. The black curves depict ray paths for the individual measurements which were averaged together to get the single kernel shown here; 52 rays were used for the zonal velocity kernel, and 39 rays - for the meridional velocity kernel. b) Selected averaging kernels for the meridional velocity inversion. The left column shows contour plots of the kernels. The contour spacing is $5 \%$ of the maximum amplitude of each kernel; dashed contours indicate a negative amplitude. The nominal locations of the three kernels, in each case marked with $\times$, are: (a) $0.72 \mathrm{R}$, (b) $0.87 \mathrm{R}$ and (c) $1 \mathrm{R} ; \lambda=$ $2.5^{\circ}$. In the right column, a cross section of each kernel is plotted, as a function of radius, at the central latitude. The arrow above each plot marks the nominal radius of the kernel; the dashed line marks the location of the centroid.

determined for the first time (Giles et al, 1997). It has a maximum speed of 20 $\mathrm{m} / \mathrm{s}$ in the subsurface layers. The flow is directed from the equator to the poles. The speed becomes smaller with depth. However, the return flow has not been detected even close to the bottom of the convection zone. However, the bottom has not been reached. Such measurements require very long ( $\sim 1$ year) time series because deep signals are much weaker than the surface or near-surface signals. The travel-time variations for the deep flows are of the order of 1 sec. So far the deep inferences have been made for 2D flows. However, this study suggests the possibility of full $3 \mathrm{D}$ imaging of the convection zone. Of course, the prime goal is to reduce the integration time. 

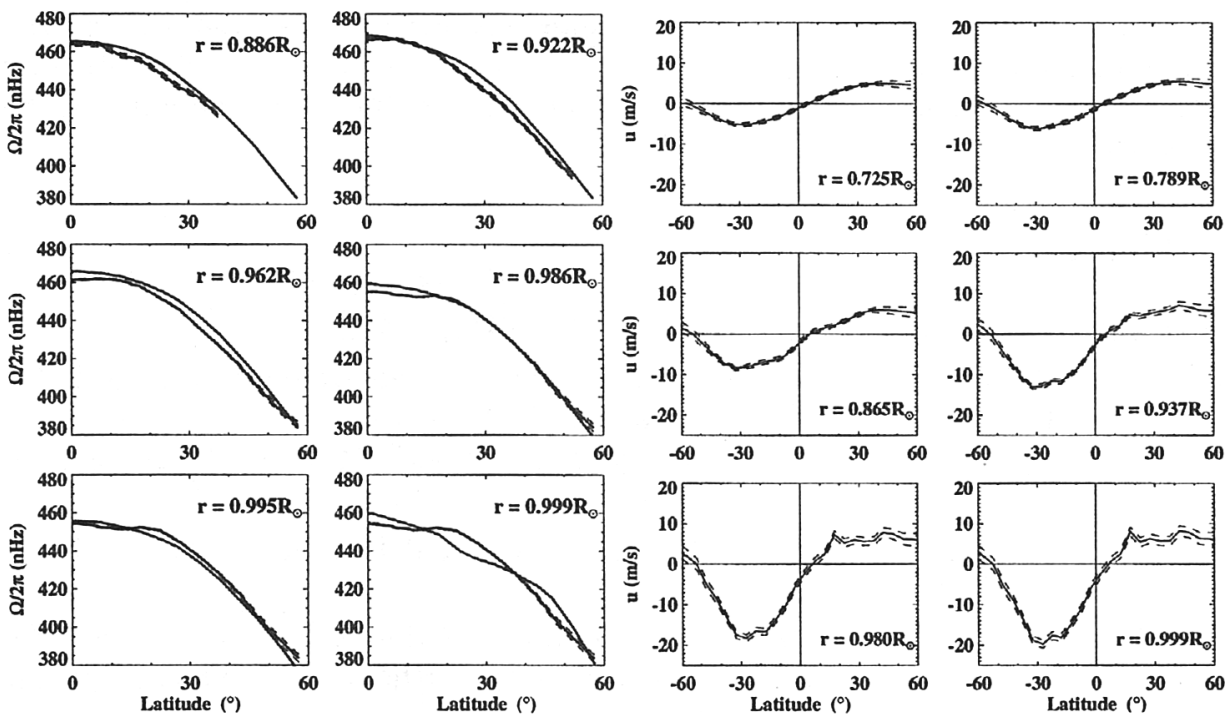

a)

b)

Figure 6. a) Comparison of the time-distance and normal mode methods for determining the solar rotation. The angular frequency is plotted versus latitude for six different choices of radial coordinate. The dotted curve is the result of an OLA inversion of frequency splittings. The solid curve is the symmetric component of the time-distance result from this work; the dashed lines are the formal errors from the inversion. b) Inversion results for the meridional circulation from 792 days of the MDI Structure Program, showing the meridional velocity as a function of latitude $\lambda$, for six different depths. Positive velocities are northward.

\section{Future Goals and Perspectives}

The unique data from SOHO/MDI have provided new views of the solar interior, and have shed light on the internal structure of sunspots and active regions, large-scale circulation and dynamics. They have allowed some new practical approaches to space weather forecasting. So far the inferences have been mostly qualitative. However, the methods of the local-area helioseismology are also being developed theoretically (e.g. Birch and Kosovichev, 2000) to fully account for the wave effects, and thus to provide more precise quantitative results.

It is now clear that for more complete understanding of the physical processes inside the Sun we need continuous high-resolution monitoring of these processes. Unfortunately, SOHO was not designed for this. This understanding and the new method have been developed in the process of the analysis of SOHO data and SOHO operation.

The work by Giles (1999) on meridional circulation was groundbreaking. There are some improvements that can be made, however. One is to use rays that penetrate below the bottom of the convection zone, which will carry a lot 
of information about the tachocline. Significant progress can be made in the study of active regions by carrying out the subsurface focusing as described by Duvall (1995). This should lead to significant improvements in resolution of the subsurface layers.

\section{References}

Birch, A.C. and Kosovichev, A.G., 2000, Solar Phys., 192, 145.

Giles, P.M. 1999, Time-Distance Measurements of Large-Scale Flows in the Solar Convection Zone, PhD Dissertation, Stanford Univ.

Giles, P.M., Duvall, T.L. Jr., Scherrer, P.H. and Bogart, R.S. 1997, Nature, 390, 52

Duvall T.L., Jr., Jefferies, S. M., Harvey, J. W., and Pomerantz, M. A., 1993, Nature, 362, 430.

Duvall T.L., Jr. 1995, in: GONG '94: Helio- and Astero-Seismology from the Earth and Space, Roger K. Ulrich, Edward J. Rhodes, Jr., Werner Däppen (eds), ASP Conf. Ser. 76, 465

Duvall T.L. and Gizon L. 2000, Solar Phys., 192, 177.

Gizon L., Duvall T.L., Jr. and Larsen R.M. 2001, These Proceedings.

Kosovichev, A.G. and Duvall, T.L., Jr. 1997, in: SCORe'96 : Solar Convection and Oscillations and their Relationship, Eds.: F.P. Pijpers, J. Christensen-Dalsgaard, and C.S. Rosenthal, Kluwer Academic Publishers (Astrophysics and Space Science Library Vol. 225),. 241

Lindsey, C. and Braun, D.C. 2000, Science, 287, 1799.

Parker, E. N. 1993, ApJ, 230, 905

Zhao, Junwei, Kosovichev, A.G. and Duvall, T.L., Jr, 2001, ApJ, submitted 